\title{
Young endothelial cells revive aging blood
}

\author{
Vivian Y. Chang, ${ }^{1}$ Christina M. Termini, ${ }^{2}$ and John P. Chute ${ }^{2,3,4}$ \\ 'Division of Hematology/Oncology, Department of Pediatrics, ${ }^{2}$ Division of Hematology/Oncology, Department of Medicine, ${ }^{3}$ Eli and Edythe Broad Center for Stem Cell Research, and ${ }^{4}$ Jonsson Comprehensive \\ Cancer Center, UCLA, Los Angeles, California, USA.
}

\begin{abstract}
The hematopoietic system declines with age, resulting in decreased hematopoietic stem cell (HSC) self-renewal capacity, myeloid skewing, and immune cell depletion. Aging of the hematopoietic system is associated with an increased incidence of myeloid malignancies and a decline in adaptive immunity. Therefore, strategies to rejuvenate the hematopoietic system have important clinical implications. In this issue of the $J C I$, Poulos and colleagues demonstrate that infusions of bone marrow (BM) endothelial cells (ECs) from young mice promoted HSC self-renewal and restored immune cell content in aged mice. Additionally, delivery of young BM ECs along with HSCs following total body irradiation improved HSC engraftment and enhanced survival. These results suggest an important role for BM endothelial cells (ECs) in regulating hematopoietic aging and support further research to identify the rejuvenating factors elaborated by BM ECs that restore HSC function and the immune repertoire in aged mice.
\end{abstract}

\section{Aging and the decline of hematologic function}

The population of the United States is inexorably aging. By 2030, between $20 \%$ and $25 \%$ of Americans will be over the age of 65 (1). Commensurate with the aging process, degenerative diseases occur with increased frequency, afflicting the neurologic, cardiovascular, and hematologic systems (2). Regenerative medicine is based on the premise that delivery of young or pluripotent stem cells can rejuvenate degenerated or exhausted organ systems. In support of this hypothesis, Castellano et al. recently demonstrated that infusions of cord blood plasma proteins revitalized the hippocampus and improved cognitive function in aged mice (3).

Aging in the hematopoietic system is characterized by hematopoietic stem cell (HSC) exhaustion, immune function decline, myeloid skewing, and an increased incidence of myelodysplasia, myeloproliferative diseases, and leukemia $(4,5)$. While allogeneic hematopoi- etic cell transplantation can cure a subset of patients with hematologic malignancies, targeted restoration of the aged bone marrow (BM) microenvironment or "niche" has not been demonstrated. As HSC self-renewal depends on paracrine signals from both the BM vascular and perivascular niches, strategies to revitalize the aging vascular niche could potentially reanimate hematopoietic system function during aging (6-9). In this issue, Poulos et al. demonstrate that infusions of young BM endothelial cells (ECs) rejuvenate the aged hematopoietic system in murine models (10).

\section{Young BM ECs support HSC function}

Poulos and colleagues demonstrate a decline in BM EC numbers, increased BM vascular leakiness, and elevated intracellular ROS levels in ECs from aged (24-month-old) C57BL/6 mice compared with those from young (3-month-old) mice. Furthermore, aged BM ECs displayed decreased prolifera-

Related Article: p. 4163

Conflict of interest: The authors have declared that no conflict of interest exists

Reference information: / Clin Invest. 2017;127(11):3921-3922. https://doi.org/10.1172/JCI97707. 
A Aged, irradiated mice

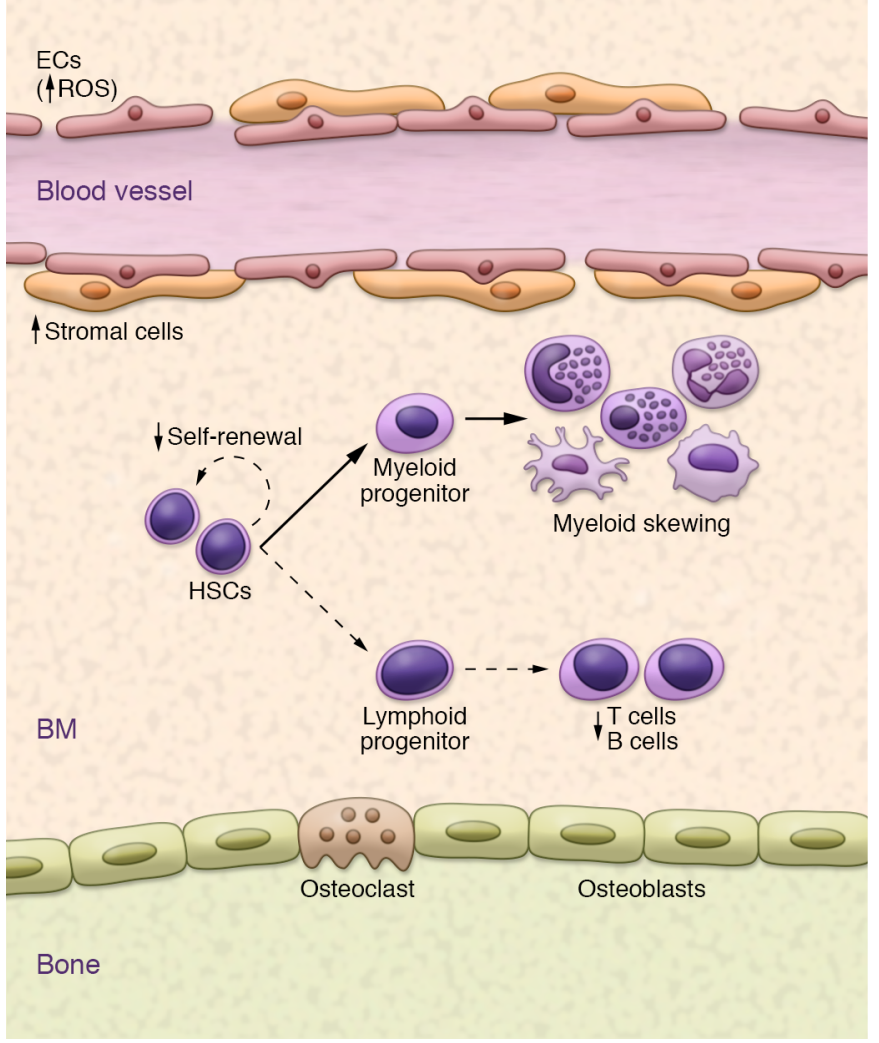

B Aged, irradiated mice + young ECs

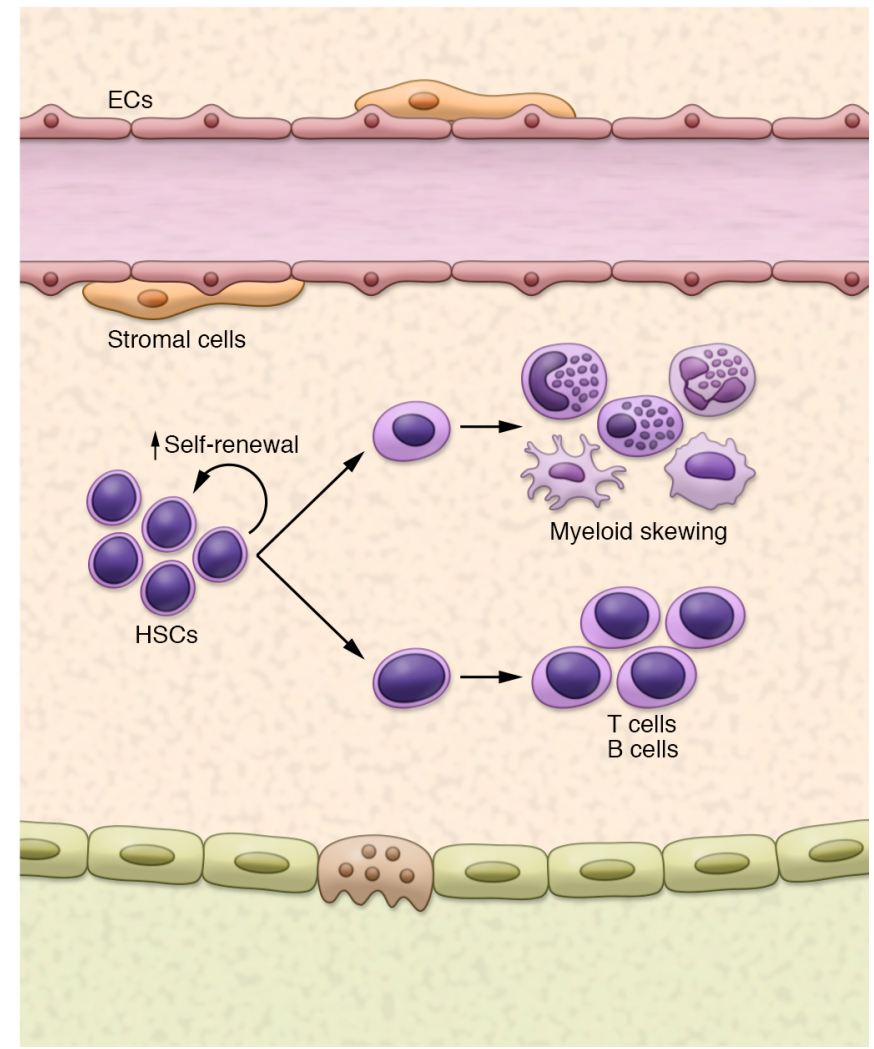

Figure 1. Infusion of BM ECs from young mice rejuvenates HSCs in aged, irradiated mice. In aged mice, there is a decline in the number of BM ECs, increased leakiness of the BM vasculature, and elevated intracellular ROS levels. The aged hematopoietic system is characterized by reduced HSC regenerative capacity, loss of T and B lymphocytes, and myeloid skewing. In this issue, Poulos and colleagues show that infusion of young ECs into aged, irradiated mice improves age-associated defects in BM ECs, restores HSC self-renewal capacity, and increases B and T cell numbers.

an aged HSC pool and hematopoietic system. These studies also highlight the translational potential of infusions of (young) ECs to accelerate hematopoietic recovery in clinically relevant scenarios. As prior studies by Poulos et al. (11) and others (12) have shown that infused ECs do not engraft in the BM vasculature, this implies that young ECs secrete soluble factors that act either directly on aged HSCs or indirectly on aged niche cells to rejuvenate the hematopoietic system. Identification of these EC-derived "fountains of youth" represents the next great challenge in understanding this process and may lead to restorative therapies that do not require cell infusion.

\section{Acknowledgments}

This work was supported in part by grant LA1 C14-08014 (to JPC) from the California Institute for Regenerative Medicine (CIRM).
Address correspondence to: John P. Chute, Division of Hematology/Oncology, Eli and Edythe Broad Center for Stem Cell Research, Jonsson Comprehensive Cancer Center, UCLA, Los Angeles, California 90095, USA. Phone: 310.206.3037; Email: jchute@mednet.ucla.edu.

1. Ortman J, Velkoff V. An aging nation: the older population in the United States. Current Populations Report. U.S. Department of Commerce, United States Census Bureau. 2014;1-28. https://www.census.gov/prod/2014pubs/ p25-1140.pdf. Accessed September 28, 2017.

2. Niccoli T, Partridge L. Aging as a risk factor for disease. Curr Biol. 2012;22(17):R741-R752.

3. Castellano JM, et al. Human umbilical cord plasma proteins revitalize hippocampal function in aged mice. Nature. 2017;544(7651):488-492.

4. Rossi DJ, et al. Cell instrinsic alterations underlie hematopoietic stem cell aging. Proc Natl Acad Sci U S A. 2005;102(26):9194-9199.

5. Pang WW, et al. Human bone marrow hematopoietic stem cells are increased in frequency and myeloid-biased with age. Proc Natl Acad Sci U S A.
2011;108(50):20012-20017.

6. Ding L, Saunders TL, Enikolopov G, Morrison SJ. Endothelial and perivascular cells maintain haematopoietic stem cells. Nature. 2012;481(7382):457-462.

7. Ding L, Morrison SJ. Haematopoietic stem cells and early lymphoid progenitors occupy distinct bone marrow niches. Nature. 2013;495(7440):231-235.

8. Greenbaum A, et al. CXCL12 in early mesenchymal progenitors is required for haematopoietic stem-cell maintenance. Nature. 2013;495(7440):227-230.

9. Poulos MG, et al. Endothelial Jagged-1 is necessary for homeostatic and regenerative hematopoiesis. Cell Rep. 2013;4(5):1022-1034.

10. Poulos MG, et al. Endothelial transplantation rejuvenates aged hematopoietic stem cell function. JClin Invest. 2017;127(11):4163-4178.

11. Poulos MG, et al. Vascular platform to define hematopoietic stem cell factors and enhance regenerative hematopoiesis. Stem Cell Reports. 2015;5(5):881-894.

12. Salter AB, et al. Endothelial progenitor cell infusion induces hematopoietic stem cell reconstitution in vivo. Blood. 2009;113(9):2104-2107. 